\title{
Biomarker quantification by multiplexed quantum dot technology for predicting lymph node metastasis and prognosis in head and neck cancer
}

\author{
Zhongliang Hu${ }^{1,2, *}$, Guoqing Qian ${ }^{1, *}$, Susan Müller ${ }^{3}$, Jing $\mathrm{Xu}^{4}$, Nabil F. Saba ${ }^{1}$, \\ Sungjin Kim ${ }^{5}$, Zhengjia Chen ${ }^{5,6}$, Ning Jiang ${ }^{1}$, Dongsheng Wang ${ }^{1}$, Hongzheng Zhang ${ }^{1}$, \\ Kristin Lane ${ }^{7}$, Clifford Hoyt', Dong M. Shin ${ }^{1}$, Zhuo Georgia Chen ${ }^{1}$ \\ ${ }^{1}$ Department of Hematology and Medical Oncology, Winship Cancer Institute, Emory University School of Medicine, Atlanta, \\ GA, USA \\ ${ }^{2}$ Department of Pathology, Xiangya Hospital, Department of Pathology, Xiangya Medical School, Central South University, \\ Changsha, Hunan, China \\ ${ }^{3}$ Department of Otolaryngology, Emory University School of Medicine, Atlanta, GA, USA \\ ${ }^{4}$ Department of Otolaryngology, Xiangya Hospital, Central South University, Changsha, Hunan, China \\ ${ }^{5}$ Biostatistics and Bioinformatics Shared Resource at Winship Cancer Institute, Emory University, Atlanta, GA, USA \\ ${ }^{6}$ Department of Biostatistics and Bioinformatics, Emory University School of Public Health, Atlanta, GA, USA \\ ${ }^{7}$ Caliper/Perkin Elmer Life Sciences and Technology, Hopkinton, MA, USA \\ *These authors are contributed equally to this work \\ Correspondence to: Zhuo Georgia Chen, email: gzchen@emory.edu \\ Keywords: multiplexed quantum dot, head and neck squamous cell carcinoma, EGFR, E-cadherin \\ Received: September 18, $2015 \quad$ Accepted: April 22, $2016 \quad$ Published: May 09, 2016
}

\section{ABSTRACT}

Purpose: To predict lymph node metastasis and prognosis in head and neck squamous cell carcinoma (HNSCC).

Results: The combination of membranous E-cadherin and membranous epidermal growth factor receptor (EGFR) quantified by QD technology with age, gender, and grade had greater predictive power than any of the single biomarkers or the two combined biomarkers quantified by conventional immunohistochemistry (IHC). The predictive power of this model was validated in another independent sample set; the predictive sensitivity of this model for LNM was $\mathbf{8 7 . 5 \%}$, with specificity up to $97.4 \%$, and accuracy $\mathbf{9 2 . 9 \%}$. Furthermore, a higher membranous E-cadherin level was significantly correlated with better overall and disease-free survival (OS, DFS; $P=\mathbf{0 . 0 0 2}, 0.033$, respectively), while lower cytoplasmic vimentin and membranous EGFR levels were significantly correlated with better OS $(P=0.016$ and 0.021 , respectively). The combined biomarkers showed a stronger prognostic value for OS and DFS than any of the single biomarkers.

Methods: Multiplexed quantum dots (QDs) were used to simultaneously label E-cadherin, vimentin, and EGFR with $\beta$-actin as an internal control. Primary tissue samples from 97 HNSCC patients, 49 with and 48 without LNM were included in the training set. Levels of membranous E-cadherin, cytoplasmic vimentin, and membranous EGFR were quantified by InForm software and correlated with clinical characteristics.

Conclusions: Multiplexed subcellular QD quantification of EGFR and E-cadherin is a potential strategy for the prediction of LNM, DFS, and OS of HNSCC patients.

\section{INTRODUCTION}

Head and neck squamous cell carcinoma (HNSCC) is the sixth most common malignancy worldwide [1]. Despite advances in understanding the molecular mechanisms of HNSCC along with improved diagnosis, the 5-year survival rate has remained virtually unchanged in the past 30 years [2,3]. Lymph node metastasis (LNM) is significantly associated with poor prognosis in HNSCC. The presence of a single ipsilateral or contralateral 
metastatic node reduces survival by $50 \%$ and bilateral disease by a further $50 \%$ [4]. Therefore, the identification of biomarkers associated with LNM could predict tumor behavior and guide treatment of HNSCC.

In one of our previous studies, we demonstrated that the subcellular localization of E-cadherin and epidermal growth factor receptor (EGFR) correlates with LNM of HNSCC [5]. It is known that epithelial-to-mesenchymal transition (EMT) is characterized by diminished epithelial features (such as loss of E-cadherin) and enhanced mesenchymal attributes (such as increased expression of cytoplasmic vimentin, fibronectin, and proteolytic enzymes). EMT has been described in embryologic morphogenesis, fibrosis and recently in tumor invasion and metastatic spread $[6,7]$. Loss of E-cadherin expression, leading to reduced intercellular adhesion, is a distinctive event in EMT and is common in metastatic carcinomas $[6,8,9]$. E-cadherin expression in HNSCC tissue specimens has been reported in several studies and is correlated with tumor progression and metastasis [5,10-13]. Cytoplasmic vimentin is considered a hallmark of mesenchymal-like conversion of epithelial cells and appears to be one of the best indicators of EMT in carcinomas including HNSCC [14-16]. EGFR is another important protein involved in progression of HNSCC. Overexpression of EGFR is observed in $80-90 \%$ of HNSCC specimens, and has been associated with a worse clinical outcome [17-20]. Therefore, these three proteins were selected as predictive biomarkers for LNM of HNSCC.

Quantum dots (QDs) are nanoscale particles made from inorganic semi-conductors. QDs have superior signal brightness, photostability, longer excited-state lifetimes, and optimized signal-to-background ratios compared with organic dyes [21, 22]. Furthermore, they have a long excitation and narrow emission spectra and can be excited simultaneously through one appropriate excitation source. Moreover, quantum dot staining is more sensitive and objective than conventional immunohistochemistry [23]. Therefore, QDs are ideal probes for both visualizing and quantifying multiple biomarkers simultaneously in the same sample.

E-cadherin, vimentin, and EGFR have all been reported to be correlated with LNM in HNSCC; however, when using one of these as a single protein marker, it is difficult to achieve both high specificity and sensitivity in the prediction of HNSCC LNM. After establishing a multiplexed QDs in head and neck cancer cell lines [24], in this study, we attempted to predict LNM using multiplexed detection of subcellular levels of the three proteins by QD technology in primary HNSCC samples. Furthermore, to minimize the effect of tissue quality on quantification of QD signals, $\beta$-actin was used as an internal control for the first time in a multiplexing system. To our knowledge, this is the first study to predict LNM using EMT-associated markers combined with EGFR using a multiplexed QD-based strategy.

\section{RESULTS}

\section{Expression of membranous E-cadherin, cytoplasmic vimentin, and membranous EGFR in tumor samples}

We initially studied a training set of primary HNSCC samples $(n=97)$. When EGFR is activated or E-cadherin is inactivated, the most obvious changes occur on the tumor cell membrane, while cytoplasmic vimentin is one of the best indicators of EMT in HNSCC. Thus, we selected membranous E-cadherin, membranous EGFR and cytoplasmic vimentin for the analysis. The levels of membranous E-cadherin were lower in tumor samples from patients with LNM [median (range) 3.54 (0.96-7.91) vs. 4.47 (1.19-21.05), $P=0.002]$; while the levels of cytoplasmic vimentin and membranous EGFR were higher in samples from patients with LNM than from those without LNM [median (range) 13.51 (6.19-34.96) vs. 10.35 (4.5-21.99), $P<0.001 ; 13.74$ (5.17-35.32) vs. 7.28 3.88-19.22), $P<0.001$, respectively] (Table 1). Moreover, tumor samples from patients with LNM showed a lower degree of differentiation than those without LNM $(P=0.004)$.

\section{Multivariate association of metastasis status with all biomarkers and covariates}

In a multivariable model after adjusting for age, gender, grade, tumor stage and the 3 biomarkers in which age, gender and grade were forced, membranous EGFR and membranous E-cadherin were significant independent predictors of LNM, respectively $(P=0.002$ for $\mathrm{E}$-cadherin and $P<0.001$ for EGFR) (Table 2).

\section{ROC analysis of predictive value of biomarkers for LNM}

To compare the power of LNM prediction using conventional immunohistochemistry (IHC) with that using QD technology, we performed ROC analyses on both previous IHC [5] and the current QD data which were generated from the same patient sample set. As shown in Table 3, for single biomarkers and the combination of biomarkers, QD analysis showed a better predictive power as compared to conventional IHC (Table 3). All 3 biomarkers were observed to have significant predictive discriminatory power for patients' LNM status. Among them, membranous EGFR had the strongest predictive discriminatory power as a single marker (Table 3 ). As shown in Table 3 and Figure 1, the combination of membranous E-cadherin and membranous EGFR had a strong predictive 
Table 1: Patient characteristics and comparison of biomarker levels between patients with and without LNM

\begin{tabular}{|c|c|c|c|c|}
\hline \multirow[b]{2}{*}{ Characteristic } & \multirow[b]{2}{*}{ Level } & \multicolumn{2}{|c|}{ Lymph Node Metastasis } & \multirow[b]{2}{*}{$P$-value* } \\
\hline & & No $N=48$ & Yes $N=49$ & \\
\hline \multirow{2}{*}{ Sex } & Male & $30(62.5)$ & $32(65.31)$ & \multirow{2}{*}{0.774} \\
\hline & Female & $18(37.5)$ & $17(34.69)$ & \\
\hline \multirow{3}{*}{ Tumor site } & Oral cavity & 24 & 26 & 0.730 \\
\hline & Oropharynx & 6 & 8 & \\
\hline & Larynx & 18 & 15 & \\
\hline \multirow{4}{*}{ Tumor size } & $\mathrm{T} 1$ & $15(31.25)$ & $13(26.53)$ & \multirow{4}{*}{0.337} \\
\hline & $\mathrm{T} 2$ & $19(39.58)$ & $17(34.69)$ & \\
\hline & $\mathrm{T} 3$ & $9(18.75)$ & $7(14.29)$ & \\
\hline & $\mathrm{T} 4$ & $5(10.42)$ & $12(24.49)$ & \\
\hline \multirow{3}{*}{ Differentiation } & WD & $14(29.17)$ & $2(4.08)$ & \multirow{3}{*}{0.004} \\
\hline & $\mathrm{MD}$ & $26(54.17)$ & $36(73.47)$ & \\
\hline & PD & $8(16.67)$ & $11(22.45)$ & \\
\hline \multirow{2}{*}{ Smoking } & No & $11(22.92)$ & $12(24.49)$ & \multirow{2}{*}{0.855} \\
\hline & Yes & $37(77.08)$ & $37(75.51)$ & \\
\hline Age & Mean $( \pm \mathrm{SD})$ & $63.13( \pm 10.97)$ & $58.73( \pm 12.84)$ & 0.074 \\
\hline Membranous E-cadherin & Median (Range) & $4.47(1.19-21.05)$ & $3.54(0.96-7.91)$ & 0.002 \\
\hline Cytoplasmic vimentin & Median (Range) & $10.35(4.5-21.99)$ & $13.51(6.19-34.96)$ & $<.001$ \\
\hline Membranous EGFR & Median (Range) & $7.28(3.88-19.22)$ & $13.74(5.17-35.32)$ & $<.001$ \\
\hline
\end{tabular}

*The $p$-value is calculated by chi-square test for sex, tumor stage, differentiation, and smoking; ANOVA for Age; Wilcoxon rank-sum test for membranous E-cadherin, cytoplasmic vimentin, and membranous EGFR.

Table 2: Best predictive model of metastasis status of patients after adjusting for 3 biomarkers and age, gender and grade (age, gender and grade were forced in the model)

\begin{tabular}{|c|c|c|c|c|c|c|}
\hline \multirow[b]{2}{*}{ Covariate } & \multirow[b]{2}{*}{ Level } & \multicolumn{5}{|c|}{ Metastasis $=$ Yes } \\
\hline & & Odds Ratio & 95\% CI Low & 95\% CI Up & OR $P$-value & Type3 $P$-value \\
\hline Age & & 0.98 & 0.93 & 1.03 & 0.333 & 0.333 \\
\hline \multirow{2}{*}{ Gender } & Male & 1.25 & 0.36 & 4.30 & 0.728 & \multirow{2}{*}{0.728} \\
\hline & Female & - & - & - & - & \\
\hline \multirow{3}{*}{ Grade } & WD & 0.10 & 0.01 & 1.03 & 0.053 & \multirow{3}{*}{0.104} \\
\hline & MD & 0.84 & 0.19 & 3.72 & 0.820 & \\
\hline & $\mathrm{PD}$ & 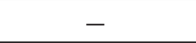 & - & - & - & \\
\hline Membrane E-cadherin & & 0.53 & 0.36 & 0.79 & 0.002 & 0.002 \\
\hline Membrane EGFR & & 1.41 & 1.19 & 1.68 & $<.001$ & $<.001$ \\
\hline
\end{tabular}

Backward selection with an alpha level of removal of .1 was used. Age, gender, and grade were forced in the model. Vimentin was removed from the model.

Hosmer and Lemeshow Goodness-of-Fit Test statistic $=4.938 ; P$-value $=0.764$ (fitted model is an adequate model). Likelihood Ratio test statistic $=64.52 ; P$-value $<0.001$ (The overall logistic regression model was significant). AUC (area under the Receiver Operating Characteristic $($ ROC) curve $)=0.919 ; P$-value $<0.001$. 
Table 3: ROC analysis of biomarkers in samples from patients with vs. without metastasis

\begin{tabular}{|c|c|c|}
\hline Biomarker & Area under ROC curve & $P$-value * \\
\hline \multicolumn{3}{|l|}{ IHC Data ${ }^{a}$} \\
\hline \multicolumn{3}{|l|}{ Single Biomarker Model: } \\
\hline Membranous EGFR & 0.6044 & 0.078 \\
\hline Membranous E-cadherin & 0.6546 & 0.009 \\
\hline \multicolumn{3}{|l|}{ Two Biomarker Model: } \\
\hline Membranous EGFR+ Membranous E-cadherin & 0.6522 & 0.010 \\
\hline \multicolumn{3}{|l|}{ Quantum Dot Data } \\
\hline \multicolumn{3}{|l|}{ Single Biomarker Model: } \\
\hline Membranous E-cadherin & 0.682 & $<.001$ \\
\hline Cytoplasmic vimentin & 0.7109 & $<.001$ \\
\hline Membranous EGFR & 0.8065 & $<.001$ \\
\hline \multicolumn{3}{|l|}{ Two Biomarker Model: } \\
\hline Membranous E-cadherin + cytoplasmic vimentin & 0.7738 & $<.001$ \\
\hline Membranous E-cadherin + membranous EGFR & 0.9009 & $<.001$ \\
\hline Cytoplasmic vimentin + membranous EGFR & 0.8104 & $<.001$ \\
\hline \multicolumn{3}{|l|}{ Three Biomarker Model: } \\
\hline Membranous E-cadherin + cytoplasmic vimentin + membranous EGFR & 0.9022 & $<.001$ \\
\hline \multicolumn{3}{|l|}{ Two Biomarker + Age+Gender+Grade Model: } \\
\hline Membranous E-cadherin + membranous EGFR + Age + Gender + Grade & 0.919 & $<.001$ \\
\hline
\end{tabular}

a: IHC data was analyzed from our publication as in reference 5. Quantum dot data was analyzed from the same specimens as in IHC data.

discrimination power (AUC: 0.9009). A model combining all three biomarkers did not further improve the prediction of LNM $(P=0.842)$, since cytoplasmic vimentin was not a significant predictor of metastasis status in the multivariable model with all 3 biomarkers (Table 2). A model combining age, gender, grade, membranous E-cadherin and membranous EGFR, shown below, displayed the best predictive power (AUC: 0.919).

$$
\begin{aligned}
& \exp (-0.0074-0.0253 \times \text { Age }+0.1098 \times \text { SexMale } \\
& +0.649 \times \text { Grade_MD }-1.4703 \times \text { Grade } \_W D \\
\hat{p}= & \frac{-0.6291 \times \text { Ecadherin }+0.3465 \times E G F R)}{1+\exp (-0.0074-0.0253 \times \text { Age }+0.1098 \times \text { SexMale }} \\
+ & 0.649 \times \text { Grade_MD }-1.4703 \times \text { Grade_WD } \\
& -0.6291 \times \text { Ecadherin }+0.3465 \times \text { EGFR })
\end{aligned}
$$

In this model, the maximized sum of sensitivity and specificity was $83.7 \%$ and $87.5 \%$, respectively, for LNM prediction when using the training set of samples (Table S1).

\section{Validation of the predictive probability model of metastasis status}

To verify the predictive probability model of metastasis status consisting of age, gender, grade, membranous E-cadherin and membranous EGFR, another independent sample set was used. As shown in Table 4, the predictive sensitivity of this model was $87.5 \%$, with specificity up to $97.4 \%$, and accuracy $92.9 \%$.

\section{Univariate survival analysis of DFS and OS}

Patients with LNM had a higher risk of death compared to those without LNM $(P=0.002)$. Older patients also had a higher risk of death $(P=0.01)$. A higher level of membranous E-cadherin was significantly correlated with better OS $(P=0.002)$, while lower levels of cytoplasmic vimentin and membranous EGFR were significantly associated with better OS $(P=0.016$ and 0.021, respectively) (Table S2). Patients with LNM had a higher risk of disease progression than those without LNM $(P=0.004)$. A higher level of membranous E-cadherin was significantly associated with a better DFS $(P=0.033)$ (Table S3). All three biomarkers were significantly correlated with OS and DFS after being dichotomized by the optimal cut-off point driven by survival analysis (Figure S1). We observed that all three biomarkers together showed a stronger prognostic value for OS and DFS than any single biomarker.

\section{Multivariable survival analysis of DFS and OS with all biomarkers and covariates}

In a multivariable model after adjusting for age, differentiation, sex, smoking history, tumor stage, LNM status and the 3 biomarkers, cytoplasmic vimentin, age, 
and LNM status were identified as significant predictors of $\operatorname{OS}(P=0.033,<0.001$, and 0.018 , respectively). Membranous E-cadherin was marginally significantly related to OS $(P=0.056)$ (Table S4). In a multivariable model after adjusting for age, differentiation, sex, smoking, tumor stage, LNM status and the 3 biomarkers, LNM status was significantly associated with DFS $(P=0.002)$ and age was marginally significantly related to DFS $(P=0.071)($ Table S5).

\section{DISCUSSION}

Accurately predicting LNM and overall prognosis in HNSCC can provide useful information for the optimization of treatment plans for patients with HNSCC as well as furthering our understanding of the biology underlying metastases. Currently, there is no standardized and consistent method to predict LNM of HNSCC using multiple protein biomarkers. In this study, we used selected EMT-related protein markers objectively quantified by QD technology to predict LNM and prognosis of HNSCC. Using a training set of HNSCC tissue samples, we initially established a LNM prediction model consisting of age, gender, grade, membranous EGFR and membranous E-cadherin. As expected, excellent levels of sensitivity $(87.5 \%)$ and specificity $(97.4 \%)$ were observed in the validation sample using the same model.

Using nanoparticle QD-based IHF, we were able to not only simultaneously quantify multiplexed biomarker levels in the same cell, but also detect their

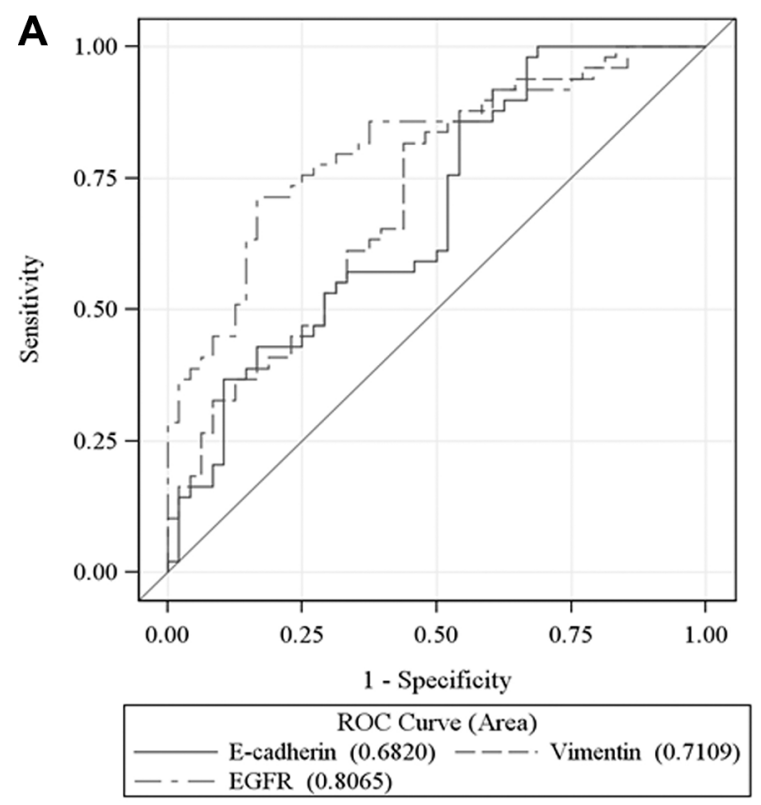

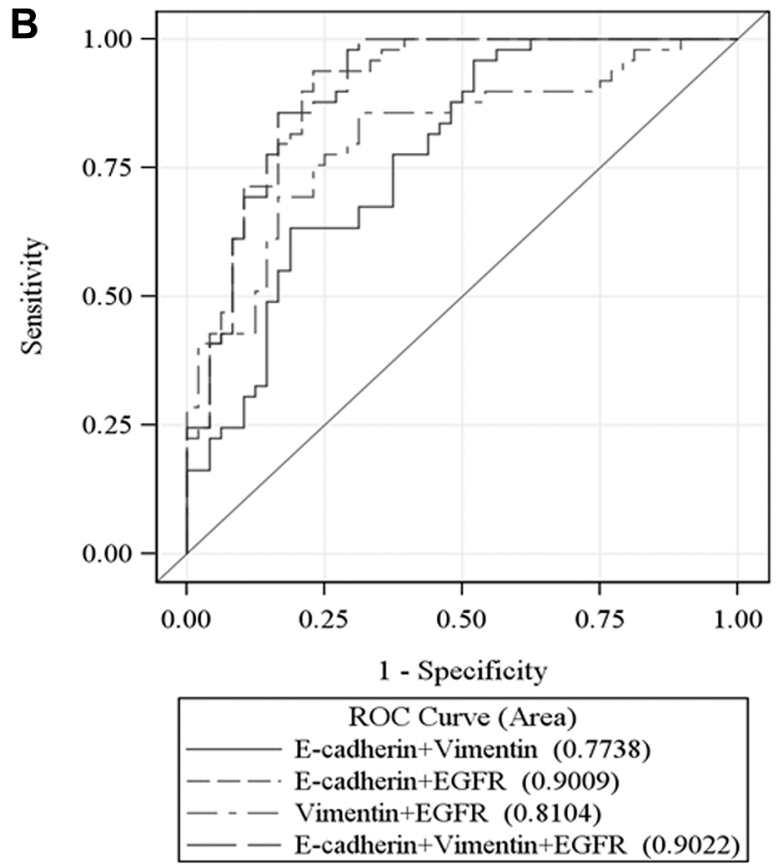

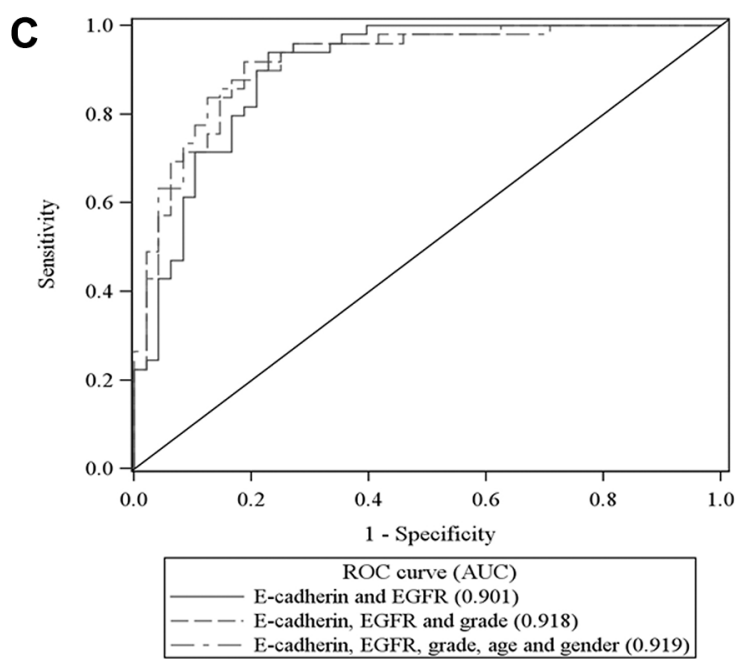

Figure 1: ROC curves for each of the 3 biomarkers (A), combined biomarkers (B), and combined biomarkers and forced age, gender and grade (C) with AUC for prediction of patient's metastasis status. 
Table 4 : Validation of the model for lymph node metastasis prediction

\begin{tabular}{|c|c|c|c|c|c|c|}
\hline \multicolumn{2}{c}{ Met } & Non-Met & \multicolumn{1}{c}{$\boldsymbol{P}$-value } & \multicolumn{1}{c}{ Sensitivity (\%) } & Specificity (\%) & Accuracy (\%) \\
\hline Prediction-Met & 28 & 1 & $<0.0001$ & 87.5 & 97.4 & 92.9 \\
\hline Prediction-Non-Met & 4 & 37 & & & & \\
\hline
\end{tabular}

levels at specific subcellular locations. Moreover, QDIHF can reveal population characteristics, such as analyte range, distribution, and variance among cells. Our study found less membranous E-cadherin, more cytoplasmic vimentin, and more membranous EGFR in primary tumor samples with LNM than in those without LNM. All three biomarkers had significant power for predicting LNM. The combination of membranous E-cadherin and membranous EGFR had stronger power to predict LNM than other combinations. Although IHC can semiquantify the subcellular levels of EGFR and E-cadherin, the LNM prediction power using IHC is much lower than using QD technology as demonstrated in the ROC analysis. Furthermore, membranous E-cadherin and EGFR together showed a stronger prognostic value for OS than any of the single biomarkers. Comparing these findings with those of our previous IHC-based study of EGFR and E-cadherin [5], QD-based IHF analysis is more objective and powerful, therefore, more accurate for prognosis of the disease. Since many proteins including EGFR and E-cadherin demonstrate dramatic changes in membrane expression when they are involved in biological activities, it is ideal to quantify the subcellular levels, rather than total, levels of these proteins. Another advantage of QD technology is that the multiplexed quantification of QD signals makes it easier to detect the staining signals of more than one protein from the nucleus, cytoplasm, or membrane of the same cell. Thus, QD-based IHF is a novel technology that uniquely addresses such issues in HNSCC biomarker studies. For QD staining, we used $\beta$-actin as an internal control for tissue quality because, as a housekeeping protein, it is constitutively expressed in tissues and we therefore quantified the biomarker levels as a value relative to $\beta$-actin levels. In our study, 3 samples were excluded because their $\beta$-actin expression was not detected. We also tested another housekeeping protein, glyceraldehyde 3-phosphate dehydrogenase (GAPDH), as a tissue quality control marker. The QD signal from GAPDH was well correlated with that from $\beta$-actin (Figure S2), suggesting that tissue quality may have a similar effect on immunostaining of any housekeeping protein which can serve as the internal control.

HPV status has been correlated with LNM [25, 26]. However, our sample set, particularly the non-metastatic group, contained few oropharyngeal tissues, which limited the analysis of HPV status as a confounding variable. Therefore, we did not consider the expression of p16, a surrogate of high-risk HPV status in the current study.
Furthermore, because our sample set included a limited number of patients with advanced HNSCC, tumor size was not observed to be significantly associated with prognosis in univariate analysis.

In summary, the subcellular expression levels of multiplexed biomarkers were assessed for the prediction of LNM using QD-based IHF technology. We found that the combination of membranous EGFR and membranous E-cadherin demonstrated strong predictive power for LNM and improved prognosis as compared to single biomarkers. Our study also demonstrated that the detection of multiplexed biomarkers using QD technology can serve as a stronger prognostic tool than a single biomarker. This method can have distinct advantages when tissue sample sizes are limited as multiple markers can be assessed simultaneously. This is a proof of principle study to demonstrate the potential use of QD-based biomarker multiplexing to establish a predictive model for LNM in head and neck cancer. Further optimization and validation of our model using prospectively recruited tumor samples is warranted in large-scale studies and the inclusion of biomarkers in combination with relevant clinical factors such as HPV status may result in establishment of a standardized method for the prediction of LNM and prognosis of HNSCC.

\section{MATERIALS AND METHODS}

\section{Patient samples}

Using an Institutional Review Board-approved consent for tissue acquisition, clinical samples for this study were obtained from surgical specimens from patients diagnosed with HNSCC from 1994 to 2003 at the Winship Cancer Institute of Emory University (Atlanta, GA). The primary treatment for these patients was surgery and no prior treatment with radiation and/or chemotherapy was administered. Patient samples consisted of a training set to establish an optimized LNM predictive model and a validation set. The training set comprised primary SCC samples from 49 patients with LNM and 48 patients without LNM, whereas the validation set consisted of 70 samples including 32 cases with LNM, and 38 without LNM. In the non-LNM group, none of the patients developed metastases within 2 years of the initial procedure. The clinical information on the samples was obtained from the surgical pathology reports in the Department of Pathology at Emory University according 
to the regulations of the Health Insurance Portability and Accountability Act. The clinicopathologic parameters for the training set, including age, sex, tobacco history, and disease stage, are characterized and listed in Table 1. Each patient's overall survival (OS) and disease-free survival (DFS) were documented through June 2012. Samples in the validation set were selected with similar anatomic locations as the training set (data not shown).

In the training set, the number of oropharyngeal samples was limited in both the non-metastasis and metastasis groups (Table 1). Therefore, the contribution of HPV status to metastasis was not studied.

\section{Quantum dot-based immunohistofluorescence (QD-IHF)}

QD-IHF for the measurement of EGFR, E-cadherin, cytoplasmic vimentin, and $\beta$-actin in tumor tissues was performed as described previously. Briefly, formalin fixed and paraffin embedded (FFPE) tissue blocks were cut into 4-5 $\mu \mathrm{m}$ sections. After deparaffinization and rehydration, antigen retrieval was performed by heating with citrate buffer $(10 \mathrm{mmol} / \mathrm{L}, \mathrm{pH} 6.0)$ in a microwave for $10 \mathrm{~min}$. The tissue slides were blocked with $10 \%$ normal goat serum for $20 \mathrm{~min}$ before the primary antibody incubation. Multiple markers were stained initially in a sequential manner with a $3 \times 5$ min phosphate-buffered saline (PBS) rinse after each step of incubation. The immunoreaction sequences were: 1) primary rabbit anti-human EGFR antibody (Biogenex, Fremont, CA), overnight at $4^{\circ} \mathrm{C}$; secondary goat anti-rabbit conjugated-QD705 (Invitrogen, Carlsbad, CA), at $37^{\circ} \mathrm{C}, 2$ hour. 2) primary mouse antihuman E-cadherin (BD Pharmingen, San Jose, CA), rabbit anti-human vimentin (Cell Signaling, Danvers, MA), and chicken anti-human $\beta$-actin (Novus Biologicals, Littleton, $\mathrm{CO}$ ) antibodies for 2 hours at room temperature; secondary goat anti-mouse conjugated-QD565, goat anti-chicken conjugated-QD655, and goat anti-rabbit conjugated-QD625 (Invitrogen) antibodies were incubated in a cocktail manner. The slides were washed three times with $1 \times$ PBS, counterstained with $4^{\prime}$,6-diamidino-2phenylindole (DAPI), mounted, and stored at $4^{\circ} \mathrm{C}$ in dark conditions. For negative controls, primary antibodies were replaced with isotype- and species-matched control antibodies.

\section{Image acquisition and unmixing}

A CRi spectral imaging system (Caliper/Perkin Elmer Life Sciences and Technology, Hopkinton, MA) with Nuance v3.1 software was used to take multiple images following the manufacturer's protocol. For each sample, a cube file consisting of serial images was acquired at $10 \mathrm{~nm}$ wavelength intervals from 425 to $720 \mathrm{~nm}$, a range covering the wavelengths of the active fluorescent QDs. To avoid variations due to cell heterogeneity, five images were randomly taken from cancerous tissue from each tissue specimen for subsequent quantification.

A spectral library for QDs 565, 625, 655, and 705 $\mathrm{nm}$ was built for deconvolution. The spectral library was then used to unmix the cube files. These images represent the distribution of each of the QDs and autofluorescence in the tissue. After the deconvolution of the images, the background signal was filtered away and only the true positive signals were shown on the images, as shown in Figure 2 .

\section{Signal quantification}

To extract the individual QD signals, the spectral library was used to unmix the imaging cube using inForm v1.4 software (Caliper/Perkin Elmer), following the manufacturer's protocol. First, a training set comprising two categories of tissue was created: cancer and stroma. The trained tissue segmentation method was used to separate cancer from its surrounding stroma until the accuracy was over $90 \%$ and differentiation of the two classes could no longer be improved. Then a builtin algorithm was used to define the membranous, cytoplasmic, and nuclear subcellular regions. Based on an analysis of images at $200 \times$ magnifications, QD fluorescence intensity in each cell was exported to an Excel spreadsheet (Microsoft, Seattle, WA) and the relative values of E-cadherin, cytoplasmic vimentin, and EGFR levels in different subcellular regions to $\beta$-actin were obtained and subjected to statistical analysis.

\section{Statistical analysis}

Three biomarkers and covariates (gender, tumor stage, differentiation, smoking history, and age) were compared between samples from patients with and without LNM using chi-square test, ANOVA (analysis of variance) or Wilcoxon rank-sum test where appropriate. Each biomarker was analyzed with logistic regression to estimate its effect on the prediction of LNM. A best predictive model was also identified by entering three biomarkers and covariates into a logistic model and using a backward variable selection method with an alpha level of removal of 0.1 . The ability of a biomarker to predict LNM status was further determined by using receiver operating characteristic (ROC) curves and measuring the area under the curve (AUC). Whether the AUCs of ROC curves were different from 0.5 (no discrimination ability) was tested with Chi-Square tests. To obtain optimal cut-off points with the best discrimination power for metastasis status, sensitivity and specificity pairs were obtained in the logistic regression under all the possible thresholds. The optimal cut-off point for each biomarker was calculated where the maximum sum of sensitivity and specificity was achieved. 
A
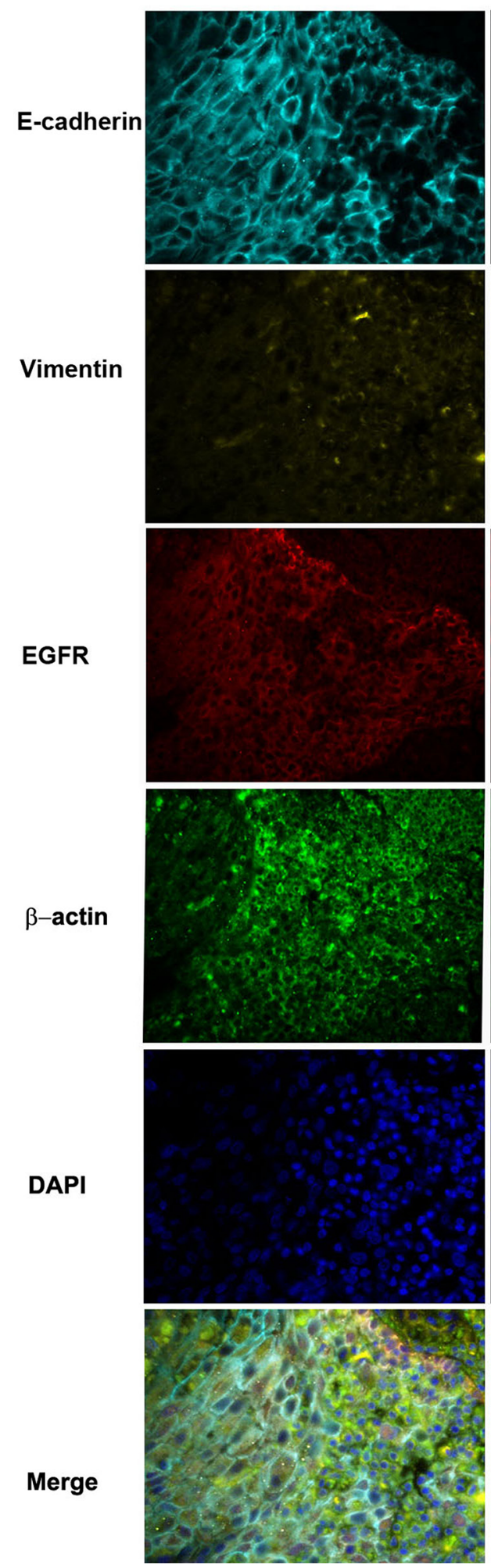

Metastasis
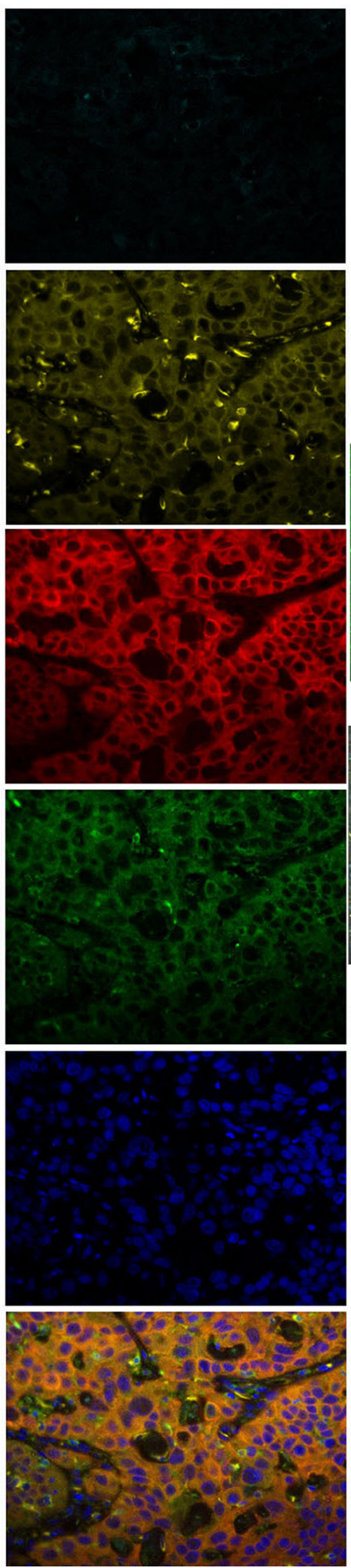

B

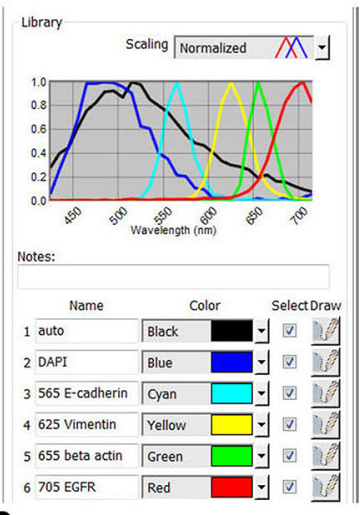

C

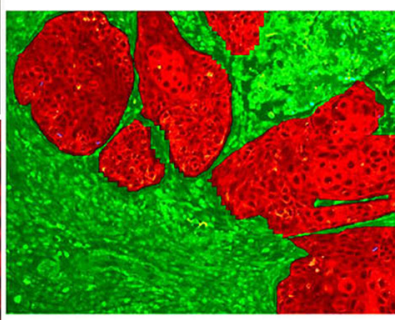

D

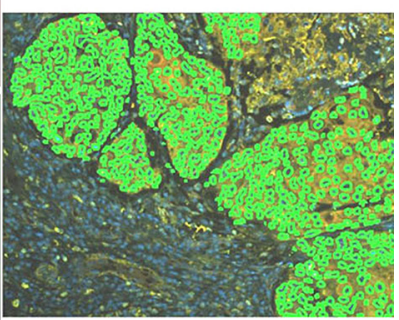

E

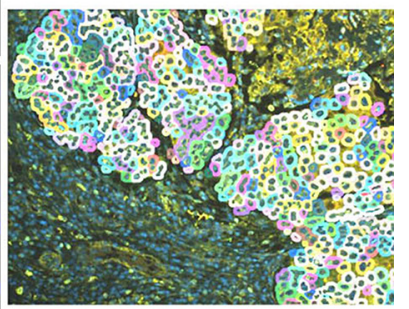

$\mathbf{F}$

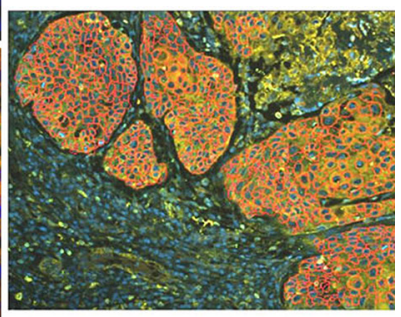

Figure 2: Simultaneous detection of three biomarkers plus control using QD-based IHF system. (A) library composed of DAPI, auto-fluorescence, QD565, QD625, QD655, and QD705 was initially set for the analysis. Signals in the image cube were unmixed according to their wavelengths in the library and then the corresponding signals were separated. E-cad is shown in cyan, EGFR in red, cytoplasmic vimentin in yellow, and $\beta$-actin in green. A: The expression of three biomarkers in primary tumors from patients with or without LNM. (B) Quantum dot library (normalized spectrum). C-F: segmentation of cancer cell from stroma (C), nucleus (D), cytoplasm (E), and membrane (F). 
Membranous E-cadherin, cytoplasmic vimentin, and membranous EGFR were further dichotomized by the optimal cut-off point, which corresponds to the most significant relationship with OS or DFS based on the log rank statistic. Cut-off values for membrane E-cadherin, cytoplasmic vimentin, and membrane EGFR were $6.57,11.92$, and 10.93, respectively. Kaplan-Meier survival estimates were then calculated for each group of patients stratified based on the optimal cut-off points along with a log-rank test. Univariate survival analysis for each covariate as well as membranous E-cadherin, cytoplasmic vimentin, and membranous EGFR was carried out using the Cox proportional hazards model. The proportional hazard assumption was also assessed. A multivariable survival analysis of membranous E-cadherin, cytoplasmic vimentin, and membranous EGFR was conducted after adjusting for gender, age, grade, and metastasis status using a backward variable selection method with an alpha level removal of 0.1 . All analyses were performed using SAS 9.3 (SAS Institute, Inc., Cary, North Carolina) and the significance level was 0.05 .

\section{ACKNOWLEDGMENTS}

We thank Dr. Anthea Hammond for editing the paper.

\section{CONFLICTS OF INTEREST}

None.

\section{FUNDING}

This study is supported by grants from the National Institutes of Health (R33 CA161873) and the Georgia Cancer Coalition Distinguished Scholar Award to ZGC.

\section{REFERENCES}

1. Haddad RI, Shin DM. Recent advances in head and neck cancer. N Engl J Med. 2008; 359:1143-1154.

2. Takes RP, Rinaldo A, Silver CE, Haigentz M, Jr., Woolgar JA, Triantafyllou A, Mondin V, Paccagnella D, de Bree R, Shaha AR, Hartl DM, Ferlito A. Distant metastases from head and neck squamous cell carcinoma. Part I. Basic aspects. Oral Oncol. 2012; 48:775-779.

3. Dasgupta S, Dash R, Das SK, Sarkar D, Fisher PB. Emerging strategies for the early detection and prevention of head and neck squamous cell cancer. J Cell Physiol. 2012; 227:467-473.

4. Richards PS, Peacock TE. The role of ultrasound in the detection of cervical lymph node metastases in clinically N0 squamous cell carcinoma of the head and neck. Cancer imaging. 2007; 7:167-178.
5. Muller S, Su L, Tighiouart M, Saba N, Zhang H, Shin DM, Chen Z. Distinctive E-cadherin and epidermal growth factor receptor expression in metastatic and nonmetastatic head and neck squamous cell carcinoma: predictive and prognostic correlation. Cancer. 2008; 113:97-107.

6. Kalluri R, Weinberg RA. The basics of epithelialmesenchymal transition. J Clin Invest. 2009; 119:1420-1428.

7. Tomaskovic-Crook E, Thompson EW, Thiery JP. Epithelial to mesenchymal transition and breast cancer. Breast cancer Res. 2009; 11:213.

8. Fidler IJ, Schackert G, Zhang RD, Radinsky R, Fujimaki T. The biology of melanoma brain metastasis. Cancer Metastasis Rev. 1999; 18:387-400.

9. Thiery JP. Epithelial-mesenchymal transitions in tumour progression. Nat Rev Cancer. 2002; 2:442-454.

10. Bowie GL, Caslin AW, Roland NJ, Field JK, Jones AS, Kinsella AR. Expression of the cell-cell adhesion molecule E-cadherin in squamous cell carcinoma of the head and neck. Clin Otolaryngol Allied Sci. 1993; 18:196-201.

11. Bosch FX, Andl C, Abel U, Kartenbeck J. E-cadherin is a selective and strongly dominant prognostic factor in squamous cell carcinoma: a comparison of E-cadherin with desmosomal components. Int J Cancer. 2005; 114:779-790.

12. Kurtz KA, Hoffman HT, Zimmerman MB, Robinson RA. Decreased E-cadherin but not beta-catenin expression is associated with vascular invasion and decreased survival in head and neck squamous carcinomas. Otolaryngol Head Neck Surg. 2006; 134:142-146.

13. Kyzas PA, Stefanou D, Batistatou A, Agnantis NJ, Nakanishi Y, Hirohashi S, Charalabopoulos K. Dysadherin expression in head and neck squamous cell carcinoma: association with lymphangiogenesis and prognostic significance. Am J Surg Pathol. 2006; 30:185-193.

14. Zeisberg $\mathrm{M}$, Neilson EG. Biomarkers for epithelialmesenchymal transitions. J Clin Invest. 2009; 119:1429-1437.

15. Dauphin M, Barbe C, Lemaire S, Nawrocki-Raby B, Lagonotte E, Delepine G, Birembaut P, Gilles C, Polette M. Vimentin expression predicts the occurrence of metastases in non small cell lung carcinomas. Lung Cancer. 2013; 81:117-122.

16. Liu LK, Jiang XY, Zhou XX, Wang DM, Song XL, Jiang HB. Upregulation of vimentin and aberrant expression of E-cadherin/beta-catenin complex in oral squamous cell carcinomas: correlation with the clinicopathological features and patient outcome. Mod Pathol. 2010; 23:213-224.

17. Ang KK, Berkey BA, Tu X, Zhang HZ, Katz R, Hammond EH, $\mathrm{Fu}$ KK, Milas L. Impact of epidermal growth factor receptor expression on survival and pattern of relapse in patients with advanced head and neck carcinoma. Cancer Res. 2002; 62:7350-7356.

18. Grandis JR, Tweardy DJ. Elevated levels of transforming growth factor alpha and epidermal growth factor receptor messenger RNA are early markers of carcinogenesis in head and neck cancer. Cancer Res. 1993; 53:3579-3584. 
19. Astsaturov I, Cohen RB, Harari P. Targeting epidermal growth factor receptor signaling in the treatment of head and neck cancer. Expert Rev Anticancer Ther. 2006; 6:1179-1193.

20. Kalyankrishna S, Grandis JR. Epidermal growth factor receptor biology in head and neck cancer. J Clin Oncol. 2006; 24:2666-2672.

21. Smith AM, Gao X, Nie S. Quantum dot nanocrystals for in vivo molecular and cellular imaging. Photochem Photobiol. 2004; 80:377-385.

22. Tholouli E, Sweeney E, Barrow E, Clay V, Hoyland JA, Byers RJ. Quantum dots light up pathology. J Pathol. 2008; 216:275-285.

23. Xu J, Muller S, Nannapaneni S, Pan L, Wang Y, Peng X, Wang D, Tighiouart M, Chen Z, Saba NF, Beitler JJ, Shin DM, Chen ZG. Comparison of quantum dot technology with conventional immunohistochemistry in examining aldehyde dehydrogenase $1 \mathrm{~A} 1$ as a potential biomarker for lymph node metastasis of head and neck cancer. Eur J Cancer. 2012; 48:1682-1691.

24. Huang DH, Su L, Peng XH, Zhang H, Khuri FR, Shin DM, Chen ZG. Quantum dot-based quantification revealed differences in subcellular localization of EGFR and E-cadherin between EGFR-TKI sensitive and insensitive cancer cells. Nanotechnology. 2009; 20:225102.

25. Joo YH, Jung CK, Sun DI, Park JO, Cho KJ, Kim MS. High-risk human papillomavirus and cervical lymph node metastasis in patients with oropharyngeal cancer. Head Neck. 2012; 34:10-14.

26. Mendelsohn AH, Lai CK, Shintaku IP, Elashoff DA, Dubinett SM, Abemayor E, St John MA. Histopathologic findings of HPV and p16 positive HNSCC. Laryngoscope. 2010; 120:1788-1794. 